\title{
ESTUDIO DE VALIDACIÓN DE LA ESCALA BEING A MOTHER \\ EN UNA MUESTRA MEXICANA DE MUJERES EMBARAZADAS
}

\section{VALIDATION STUDY OF THE BEING A MOTHER SCALE IN A}

MEXICAN SAMPLE OF PREGNANT WOMEN

Mónica Reya Martínez, Minerva T. Vanegas Farfano, Mónica T. González Ramírez

Universidad Autónoma de Nuevo León, México

Correspondencia:monica.reymar@gmail.com

\section{RESUMEN}

Antecedentes: La insatisfacción con la experiencia del embarazo, puede traer repercusiones emocionales en las mujeres por influencia de factores cognitivos y psicosociales en torno al rol materno y la etapa del embarazo. Objetivo: Evaluar la estructura factorial de la escala Being a Mother en su adaptación al español, como medida de la experiencia emocional del embarazo en una muestra de mujeres mexicanas embarazadas. Método: Participaron 300 mujeres embarazadas, pertenecientes a clínicas públicas del sector salud. Los datos se examinaron mediante el Análisis Factorial Exploratorio, usando el Análisis Paralelo y rotación Promim, evaluando los índices de bondad de ajuste mediante el método de Hull. Resultados: La consistencia interna es de $\alpha=.88$, con evidencia de validez de criterio evaluada con medidas de estrés $\left(\mathrm{r}_{\mathrm{s}}=.484\right)$ y autoeficacia $\left(\mathrm{r}_{\mathrm{s}}=\right.$ -.309) resultando significativas $(\mathrm{p}=.001)$. Se determinó una estructura unidimensional que explica el $74.65 \%$ de la varianza, con adecuada validez convergente $(\mathrm{AVE}>.5)$, e índices de bondad de ajuste apropiados (RMSEA 
$=.062, \mathrm{CFI}=.99, \mathrm{AGFI}=.99)$. Conclusiones: Se aporta un instrumento con adecuadas propiedades psicométricas para evaluar la satisfacción de la experiencia emocional del embarazo y el rol materno en mujeres.

Palabras clave: experiencia emocional del embarazo; autoeficacia; estrés; adaptación.

\section{ABSTRACT}

Background: Dissatisfaction with the pregnancy experience can bring emotional repercussions on women due to the influence of cognitive and psychosocial factors around the maternal role and the stage of pregnancy. Objective: To evaluate the factorial structure of the Being a Mother scale in its spanish adaptation, as a measure of the emotional experience of pregnancy in a sample of pregnant Mexican women. Method: Three hundred pregnant women participated, belonging to public health sector clinics. The data was examined by the Exploratory Factor Analysis, using Parallel Analysis and Promim rotation, evaluating the goodness of fit indexes by the Hull method. Results: The internal consistency is $\alpha=$ .88 , with evidence of criterion validity evaluated with measurements of stress $\left(\mathrm{r}_{\mathrm{s}}=.484\right)$ and self-efficacy $\left(\mathrm{r}_{\mathrm{s}}=-.309\right)$ resulting significant $(\mathrm{p}=$ $.001)$. A one-dimensional structure was determined that explains $74.65 \%$ of the variance, with adequate convergent validity (AVE $>.5$ ), and appropriate goodness of fit indexes $(\mathrm{RMSEA}=.062, \mathrm{CFI}=.99, \mathrm{AGFI}=$ .99). Conclusions: An instrument with adequate psychometric properties is provided to evaluate the satisfaction with the emotional experience of pregnancy and the maternal role in women.

Key words: emotional experience of pregnancy; self-efficacy; stress; validation; adaptation. 


\section{INTRODUCCIÓN}

El embarazo es un suceso vital estresante (Sandín \& Chorot, 2017) donde la mujer presenta modificaciones biológicas para ajustarse al desarrollo del feto (Soma-Pillay et al., 2016) y denota su nivel de madurez psicológica para adaptarse y enfrentar cambios promovidos en la etapa (Gómez \& Aldana, 2007). Se ha reportado que durante este proceso, participan recursos internos y externos, así como evaluaciones cognitivas, que si son funcionales permiten valorar el evento como un desafío y gozar de un mayor sentido de crecimiento personal (Taubman-Ben-Ari et al., 2009).

Cuando la mujer no afronta favorablemente la etapa, experimenta secuelas psicológicas tales como depresión y ansiedad (Ajinkya et al., 2013) esto, al sobreañadirse un proceso de estrés como respuesta a estímulos estresantes (Valdés \& García-Esteve, 2017). Ello, conduce a la adopción de conductas de riesgo y a una disminución en las habilidades para atender las demandas implicadas, repercutiendo tanto en la madre como el feto (Lara \& Navarrete, 2012). Así, resulta importante conocer el sentido de autoeficacia, al tratarse de un recurso cognitivo que puede afectar la cantidad de estrés, depresión y niveles de motivación frente a situaciones amenazantes o difíciles (Bandura, 1989).

Aunado a lo anterior, se destaca el estudio de la experiencia emocional durante el embarazo, porque denota las evaluaciones cognitivas implicadas en la identificación con la etapa, manifestado en el grado de compromiso con el desarrollo fetal y los comportamientos ante las demandas del embarazo (Camberis et al., 2014). En este sentido, Condon (1993) enfatiza la evaluación de aspectos subjetivos en mujeres embarazadas mediante instrumentos fiables y válidos que puedan utilizarse como herramientas de cribado clínico en relación al apego, actitudes o comportamientos de 
riesgo para el feto, así como la manifestación de síntomas psicológicos. Lo cual implica que los cuestionarios posean como base un marco teórico, que contribuyan con la elaboración de explicaciones y predicciones de una forma coherente. Con ello, además de indagar las probables causas del desencadenamiento de sintomatología emocional desde el embarazo, se puede conseguir la implementación de estrategias para prevenir la cronicidad de padecimientos psicológicos (Lara et al., 2016) ya que si no se tratan a tiempo pueden extenderse al postparto (Chen et al., 2019; Navarrete et al., 2012; Parfitt \& Ayers, 2014).

\section{La escala Being a Mother (BaM-13)}

Existen instrumentos centrados en la teoría de la autoeficacia de Bandura que miden la confianza de los padres durante la crianza de los hijos (Crncec et al., 2010). Matthey (2011) desarrolló la escala Being a Mother (BaM-13, por sus siglas en inglés) como medida breve de autoinforme que considera aspectos generales de la autoeficacia, pero integra la experiencia de la maternidad en mujeres con hijos recién nacidos hasta preescolares. Cubre temas coherentes con la salud mental perinatal, destacándose el aislamiento social, culpa, relación con el hijo, arrepentimiento, afrontamiento, autoconfianza, y satisfacción con el apoyo social. Se excluyen áreas referentes con el sueño, la alimentación y otros roles (pareja, amiga, hija, etc.). En esta forma, el estudio determinó su sensibilidad clínica para identificar a mujeres con dificultades en su rol e insatisfacción con la vivencia de la maternidad (Crncec et al., 2010; Matthey, 2011).

En su estudio, Matthey (2011) evaluó la fiabilidad mediante el alfa de Cronbach $(\alpha=.79)$; obteniendo además una fiabilidad test-retest con una correlación moderada de .52 en un intervalo de tiempo de 6 semanas, mientras que en un lapso de 2 a 3 semanas fue de .74. Asimismo utilizó la 
correlación inter-ítem, mostrándose ausencia de redundancia en los ítems. El análisis factorial arrojó tres factores que explicaron el 54.9\% de la varianza, los cuales son: experiencia infantil, que consiste en la experiencia de la madre con el niño (ítems 3, 9, 10, 12, 13); experiencia adulta, centrado en las experiencias de la mujer como adulta (ítems 2, 5, 6, 7, 8, 11); y cercanía emocional con el bebé o niño, centrado en la vinculación emocional (ítems 1 y 4). La validez concurrente, se obtuvo mediante la Escala de Depresión Posnatal de Edimburgo (Cox et al., 1987) (EPDS, por sus siglas en inglés), obteniéndose una correlación de .64 e indicando que las escalas miden construcciones diferentes. La validez discriminante fue evaluada con las escalas Reason for Postnatal Distress ChecklistBrief (RDP-b) (Matthey, 2009), el Communication, Understanding, Relationship, and Personality Questionnaire (CUR-P) realizado por el autor; la medida Current mood (Matthey, 2009) y The Karitane Parenting Confidence Scale (KPCS) (Crncec et al., 2008). Los resultados mostraron diferencias estadísticamente significativas entre la BAM-13 y medidas de personalidad confiada $(\mathrm{p}=<.001 ; \mathrm{d}=.95)$, personalidad preocupante $(\mathrm{p}$ $=<.001 ; \mathrm{d}=.84)$, apoyo de pareja $(\mathrm{p}=<.001 ; \mathrm{d}=1.25)$ y calidad de la relación de pareja $(\mathrm{p}=<.001 ; \mathrm{d}=1.45)$, indicadas anteriormente.

Si bien, el estudio de Matthey (2011) contempla una muestra de madres con hijos recién nacidos hasta preescolares, acentúa la importancia de identificar las evaluaciones que hace una mujer sobre la experiencia de la maternidad y el rol materno, lo que brinda información sobre su nivel de satisfacción o disfrute, sin limitarse a la medición del estado de ánimo o la detección de la depresión como lo hace el EPDS. A saber, existen escalas dirigidas a la medición de la depresión, autoeficacia relacionada con las edades de los hijos y otras variables en las madres durante la crianza y el postparto (Crncec et al., 2010; Jadresic, 2014; Matthey, 2011). 
No obstante, existe necesidad de instrumentos que evalúen la percepción de las mujeres sobre la vivencia del embarazo, que de ser insatisfactoria, permita a los especialistas de la salud una exploración más extensa sobre los factores psicosociales que influyen en ello. Lo anterior, porque se tiene conocimiento que las valoraciones cognitivas son fundamentales para los juicios e interpretaciones y pueden influir en las reacciones emocionales (Bandura, 1989) creencias y consecuentemente en los comportamientos en un momento determinado (Olaz, 2003). Por lo cual, evaluar la estructura factorial del presente instrumento en su adaptación al embarazo, puede permitir obtener una medida útil que proporcione información acerca de la vivencia y dificultades por las que puede pasar una mujer en dicha etapa. En resumen, con estos antecedentes se pretende evaluar la estructura interna de la escala BaM-13 adaptada para mujeres mexicanas embarazadas, como medida de la percepción y satisfacción de la experiencia del embarazo y el rol materno.

\section{MÉTODO}

\section{Diseño}

El estudio es instrumental, de tipo transversal; utilizado para analizar las propiedades psicométricas de instrumentos, evaluadas en un momento temporal sin pretender relaciones causa-efecto (Ato et al., 2013).

\section{Participantes}

El muestreo fue no aleatorio por conveniencia, conformándose por casos que se encontraban en el lugar de la aplicación (Ato et al., 2013). Se tomó en cuenta la inclusión de un mínimo de 200 observaciones para favorecer la estabilidad de la solución factorial (Ferrando \& Anguiano-Carrasco, 2010), quedando en un tamaño muestral de 300 mujeres mexicanas embarazadas pertenecientes a un programa de aseguramiento voluntario, brindado por una institución pública de salud al carecer de seguridad social. 


\section{Instrumentos}

Historia sociodemográfica e historia clínica y obstétrica. Se desarrolló un cuestionado ad hoc para obtener información socioeconómica, obstétrica y de salud mental.

Escala Being a Mother (BaM-13, Matthey, 2011). Escala de tipo Likert que mide la experiencia emocional y la satisfacción con el rol materno en las últimas 2 a 3 semanas. Posee 13 ítems para responderse mediante 4 puntos de respuesta que van de 0 a 3 dependiendo de la dirección de la pregunta. Estas opciones son: Sí, la mayoría o todo el tiempo; Sí, algunas veces; No, no muy a menudo y No, rara vez o nunca. Las calificaciones se acomodan en el siguiente orden $0,1,2$ y 3 , con el fin de minimizar la confusión y el error de puntuación ante escalas con reactivos inversos. Su recorrido es de 0 a 39, donde puntuaciones altas indican menor satisfacción con la experiencia de la maternidad. Contiene una pregunta cualitativa ( $\mathrm{Si}$ has sentido que ser madre es muy estresante, muy difícil, o desagradable ¿A qué crees que se deba?), que durante el estudio original permitió establecer el punto de corte de 9 , el cual fue calculado tomando en cuenta el estrés y no criterios diagnósticos para la depresión. El autor estableció que a partir de dicho valor, la madre se encontraba más estresada e insatisfecha con la experiencia de maternidad y/o el rol.

Escala general de autoeficacia (Schwarzer \& Jerusalem, 1995). Se utilizó la versión adaptada al español (Baessler \& Schwarcer, 1996), que consiste en una escala tipo Likert y mide la competencia personal frente a demandas difíciles o creencias optimistas de que las conductas son responsables de los resultados exitosos. Contiene 10 ítems y las respuestas van de 4 (muy cierto) a 1 (incorrecto). No cuenta con punto de corte, pero puntuaciones altas indican mayor autoeficacia. El alfa de Cronbach es de .79 a .93. 
Estrés percibido (PSS; Cohen et al., 1983). Se utilizó la escala tipo Likert validada en México (González \& Landero, 2007). Mide el estrés percibido mediante 14 ítems, 7 de ellos se califican directamente y los restantes en orden inverso. Las respuestas van de 0 (nunca) a 4 (muy a menudo), donde a mayor puntuación mayor estrés percibido. Su alfa de Cronbach es de .83 .

\section{Procedimiento}

El presente estudio fue aprobado en sus aspectos éticos y metodológicos por el Comité Doctoral de la Facultad de Psicología de la Universidad Autónoma de Nuevo León. Para la aplicación de los instrumentos se tuvo el consentimiento de las autoridades correspondientes, en este caso, el subdirector de Enseñanza e Investigación de la Secretaría de Salud del Estado de Coahuila, México, así como el director de la Jurisdicción Sanitaria de la entidad. De esta manera, se accesó a población de mujeres embarazadas en diversas clínicas de la institución para la recogida de datos, contemplándose criterios éticos propuestos en la Declaración de Helsinki (World Medical Association, 2013) y por la Asociación Americana de Psicología (2017).

En primer lugar, para la adaptación del instrumento se adoptó la estrategia de traducción directa y el análisis de equivalencia (Carretero-Dios \& Pérez, 2005), dos expertos tradujeron los ítems al español y dos expertos en el tema revisaron la equivalencia conceptual. Ante el objetivo de evaluar la experiencia emocional durante el embarazo, las instrucciones se dispusieron para responder las afirmaciones pensando en las últimas 2 o 3 semanas, conservando el mismo lapso de tiempo que la versión original, con el fin de evitar que la mujer pensara en eventos específicos estresantes de la última semana (Matthey, 2011). 
Posteriormente se realizó el estudio piloto en el que participaron 35 mujeres embarazadas, quienes acudían a un consultorio particular de la localidad saltillense y que aceptaron participar voluntariamente, y para lo cual firmaron un consentimiento informado. Se obtuvo un alfa de Cronbach de .82 considerado como buena consistencia interna (Hair et al., 2014). Dentro de las características generales de las participantes, fueron una edad media de 25.37 años ( $\mathrm{DE}=4.82)$, el 60\% con escolaridad secundaria, el 74\% viviendo en unión libre, 91\% dedicadas a labores domésticas, $83 \%$ sin complicaciones obstétricas. Además respecto al padecimiento de trastornos psiquiátricos, el $97 \%$ no contó con diagnóstico previo al embarazo, mientras que la totalidad de las mujeres no había recibido algún diagnóstico durante la gestación, al momento del estudio.

Finalmente, se hizo la colecta de datos en las instituciones de salud pública, en donde igualmente se brindó un consentimiento informado por escrito que fue explicado verbalmente, y después de aceptar su participación voluntaria fue firmado por las participantes. Al terminar se les retroalimentó verbalmente sobre los resultados, se les entregó un folleto con información relativa a salud mental materna y fueron orientadas sobre centros especializados para la atención psicológica de bajo costo y gratuita.

En este caso las participantes contaban una edad promedio de 21.62 (DE $=4.6$ ) con un rango de 17 a 42 años; contaban con educación secundaria $(67.3 \%)$, vivían en unión libre (73.7\%), se dedicaban a labores domésticas $(79.7 \%)$ y percibieron subjetivamente un nivel socioeconómico medio (78\%). El 66.3\% $(n=199)$ eran primigestas y el 33.7\% $(n=101)$ multigestas. De éstas, $18 \%$ se encontraban en el primer trimestre de embarazo, 34\% en el segundo y $48 \%$ en el tercero. Un 20\% informó haber experimentado complicaciones obstétricas (amenaza de aborto, preeclampsia, anemia, 
ascos, bajo peso, cansancio, dolores abdominales y corporales). El 14\% contaba con antecedentes familiares de depresión y el 9.3\% de ansiedad; asimismo el 1\% fue diagnosticada con ansiedad y depresión durante el embarazo.

\section{Análisis de datos}

Se utilizó el paquete estadístico para las ciencias sociales IBM SPSS versión 24 para estimar los datos descriptivos, la consistencia interna mediante el análisis del alfa de Cronbach. El ajuste de normalidad se evaluó con la prueba Kolmogorov-Smirnov, optando por efectuar correlaciones de Spearman para evaluar la validez de criterio. Como criterios de oro, se eligieron tanto la escala de autoeficacia y de estrés percibido, ya que por un lado Matthey (2011) tomó en consideración aspectos generales de la autoeficacia al desarrollar la escala BaM-13, y por otro, el punto de corte que estableció va en función del estrés.

La estructura interna se evaluó con el AFE mediante el programa Factor (Lorenzo-Seva \& Ferrando, 2013) en su versión10.9.02. Dicho software, es de libre acceso para la ejecución de análisis factoriales exploratorios y también provee soluciones semiconfirmatorias. Tiene disponible diversos procedimientos de rotación (ortogonales y oblicuos), de estimación (mínimos cuadrados no ponderados, el análisis paralelo, el método de Hull, etc.), igualmente ofrece estadísticas descriptivas univariantes y multivariantes, medidas de adecuación e índices de bondad de ajuste.

El análisis de datos se basa en la matriz de correlaciones policóricas para ítems politómicos (Lloret-Segura et al., 2014), los cuales fueron analizados para determinar la varianza no común explicada que corresponde al error no atribuible a los factores (Lloret-Segura et al., 2014; Timmerman \& 
Lorenzo-Seva, 2011). El número de factores se calculó por el método de Análisis Paralelo y rotación Promin. Se estimaron índices de bondad de ajuste a través del Método de Hull, ya que es una técnica que ubica el punto en el cual la bondad de ajuste y la parsimonia se equilibran óptimamente (Lorenzo-Seva et al., 2011).

\section{RESULTADOS}

La Tabla 1 muestra los ítems del instrumento, mientras que la Tabla 2 contiene los estadísticos descriptivos y de consistencia interna de las variables, los cuales son mayores a .70 considerados como adecuados, ya que el límite inferior acordado en el alfa de Cronbach es $\geq .70$, pudiendo disminuir a $\alpha=.60$ en investigaciones exploratorias (Hair et al., 2014). 
Tabla 1

Traducción y Adaptación de la Escala Being a Mother

1. I have felt confident about looking after my baby/toddler

2. I have missed the life I had before I became pregnant with this baby/toddler (or for adoptive mothers: before I had this baby/toddler)

3. I have found it hard to cope when my baby/toddler cries

4. I have felt close to my baby/toddler

5. I have felt lonely or isolated

6. I have felt bored

7. I have felt unsupported

8. I have felt alright about asking people for help or advice when I needed to

9. I have felt nervous or uneasy around my baby/toddler

10. I have been worried that something would happen to my baby/toddler

11. . I have been annoyed or irritated with my baby/toddler

12. I worry I am not as good as other mothers

13. I have felt guilty

If you have found being a mother very stressful, very difficult, or unenjoyable, why do you think this is?
1. Me he sentido confiada al cuidar mi embarazo

2. He extrañado la vida que tenía antes de embarazarme del bebé

3. Me ha costado trabajo lidiar o sobrellevar el embarazo

4. Me he sentido muy cercana o apegada a mi bebé

5. Me he sentido sola o aislada durante el embarazo

6. Me he sentido aburrida durante el embarazo

7. Me he sentido sin apoyo durante el embarazo

8. Me he sentido bien al pedir ayuda o consejos a la gente por el embarazo cuando lo he necesitado

9. Me he sentido nerviosa o intranquila por el embarazo

10. He estado preocupada de que algo le fuera a pasar a mi bebé en el embarazo

11. He estado molesta o irritada por el embarazo

12. Me preocupa no ser tan buena como otras madres

13. Me he sentido culpable por el embarazo

Si has sentido que ser madre es muy estresante, muy difícil, o desagradable ¿A qué crees que se deba?

Nota: Esta tabla contiene los reactivos de la escala original Being a Mother (Ser madre), y en español en su versión final.

Tabla 2

Datos Descriptivos y Alfa de Cronbach de las Escalas

\begin{tabular}{l|c|c|c|c|c|c|c}
\hline \multicolumn{1}{c|}{ Escala } & Alfa de Cronbach & Media & Mediana & DE & Mínimo & Máximo & Ítems \\
\hline Escala ser madre $^{\mathrm{a}}$ & .83 & 12.70 & 11 & 7.73 & 0 & 31 & 13 \\
Escala ser madre $^{\mathrm{b}}$ & .88 & 9.40 & 8 & 7.20 & 0 & 26 & 9 \\
Estrés percibido & .74 & 19 & 19 & 7.28 & 1 & 44 & 14 \\
Autoeficacia & .89 & 32.69 & 34 & 6.43 & 12 & 40 & 10
\end{tabular}

Nota: $\mathrm{a}=$ los datos descriptivos corresponden a la escala con 13 ítems. $\mathrm{b}=$ los datos descriptivos corresponden a la escala de 11 ítems. DE = desviación estándar. 
La prueba Kolmogorov-Smirnov examinó la normalidad, obteniéndose valores significativos menores a .05 para experiencia del embarazo $(\mathrm{p}=$ $.001)$, autoeficacia $(p=.001)$ y estrés percibido $(p=.003)$. La evidencia de validez de criterio, se determinó mediante correlaciones de Spearman, siendo positiva y significativa entre experiencia del embarazo y estrés $\left(\mathrm{r}_{\mathrm{s}}\right.$ $=.48, \mathrm{p}=.001)$, lo que indica que cuando una variable se incrementa la otra también (Clark-Carter, 2002), es decir, que a mayor insatisfacción con la experiencia del embarazo mayor estrés. Asimismo, se arrojó una correlación negativa y significativa entre experiencia del embarazo y autoeficacia $\left(\mathrm{r}_{\mathrm{s}}=-.30, \mathrm{p}=.001\right)$, indicativo de que cuando una variable disminuye la otra aumenta (Clark-Carter, 2002), así, a mayor insatisfacción con la experiencia del embarazo menor autoeficacia, o por el contrario, a mayor autoeficacia menor insatisfacción.

El análisis de confiabilidad y homogeneidad, indica adecuada variabilidad de los ítems 2, 3, 5, 6, 7, 9, 10, 11, 12 y 13, al mostrarse desviaciones estándar cercanas a 1 y mayores a dicho valor (Carretero-Dios \& Pérez, 2005). Además, como criterio de confiabilidad se observa que si se elimina cualquiera de los ítems del instrumento, la consistencia interna es buena y no disminuye por debajo de $\alpha=.80$. Sin embargo, la correlación total de elementos corregida es inferior a .20 en los ítems 1, 4, 8 y 10, lo que denota baja correlación entre las preguntas (Nunnally, 2009) y puede ser indicativo de que están midiendo un constructo distinto o poseen escasa relevancia dentro de la escala (Frias-Navarro, 2020) dirigida a mujeres embarazadas. Al observarse curtosis positivas $>2$, se opta por eliminar los ítems 1 y 4, pero en el análisis factorial se conservan los ítems 8 y 10 con el fin de evaluar su desempeño y corroborar si se descartan (Tabla 3). 


\section{Tabla 3}

Descriptivos de los Ítems, Correlación Total de Elementos Corregida y Consistencia Interna al Suprimir Elementos

\begin{tabular}{|c|c|c|c|c|c|c|}
\hline İtem & Media & $\overline{D E}$ & Curtosis & Asimetría & $\begin{array}{l}\text { Correlación total de } \\
\text { elementos corregida }\end{array}$ & $\begin{array}{l}\text { Alfa de Cronbach si se } \\
\text { suprime elemento }\end{array}$ \\
\hline 1 & .46 & .73 & 3.11 & 1.78 & .18 & .84 \\
\hline 2 & 1.14 & 1.06 & -1.21 & .32 & .56 & .81 \\
\hline 3 & .96 & 1.11 & -1.05 & .65 & .68 & .80 \\
\hline 4 & .47 & .79 & 2.60 & 1.78 & .14 & .84 \\
\hline 5 & 1.01 & 1.14 & -1.16 & .60 & .72 & .80 \\
\hline 6 & .98 & 1.07 & -1.04 & .59 & .66 & .81 \\
\hline 7 & .85 & 1.16 & -.70 & .96 & .68 & .80 \\
\hline 8 & .62 & .79 & 1.45 & 1.32 & .07 & .84 \\
\hline 9 & 1.36 & 1.02 & -1.16 & .02 & .43 & .82 \\
\hline 10 & 1.73 & .95 & -.61 & -.51 & .10 & .84 \\
\hline 11 & 1.24 & 1.06 & -1.28 & .15 & .60 & .81 \\
\hline 12 & 1.16 & 1.11 & -1.30 & .33 & .55 & .81 \\
\hline 13 & .67 & 1.16 & -.023 & 1.34 & .65 & .81 \\
\hline
\end{tabular}

Nota: DE = Desviación estándar.

\section{Análisis factorial exploratorio}

Se evaluó el coeficiente Kaiser-Mayer-Olkin $(\mathrm{KMO}=.890)$ y la prueba de esfericidad de Bartlett ( $\mathrm{p}<.05=.001$ ), ambos considerados adecuados. Con base en ello, se efectuó el AFE por Análisis Paralelo y rotación Promin, estimándose la matriz de correlación policórica por la naturaleza politómica de las variables. Las cargas factoriales fueron menores a .40 en los ítems 8 y 10, y tras su eliminación se sugirió un factor que explica el $74.65 \%$ de la varianza (Tabla 4 ). 


\section{Tabla 4}

Cargas Factoriales y Comunalidades Compartidas de los ítems

\begin{tabular}{cccc}
\hline Ítem & Carga factorial & Comunalidad \\
\hline 2 & .69 & .48 \\
3 & .82 & .67 \\
5 & .85 & .73 \\
6 & .79 & .63 \\
7 & .89 & .80 \\
8 & .07 & .00 \\
9 & .50 & .25 \\
10 & .08 & .00 \\
11 & .71 & .50 \\
12 & .63 & .40 \\
13 & .89 & .80 \\
\hline
\end{tabular}

Nota: Las cargas factoriales son menores a .40 en los ítems 8 y 10.

Al evaluarse el factor unidimensional mediante el Método de Hull con extracción de Mínimos Cuadrados no Ponderados Robustos (RULS), se observó un incremento en la prueba de Kaiser-Meyer- Olkin (KMO $=.90$ ), así como adecuada validez convergente observada en la varianza media extraída (AVE) de .75 (> .50) (Hair et al., 2014). Aunado a ello, se consideraron parámetros propuestos por $\mathrm{Hu}$ y Bentler (1999) para determinar la bondad de ajuste, obteniéndose un valor cercano a lo esperado en el error cuadrático de aproximación > .06 (RMSEA = .06), un índice de ajuste comparativo $(\mathrm{CFI}=.99)$ e índice no normalizado de ajuste $(\mathrm{NNFI}=.98)$ mayores a .90. El estadístico de bondad de ajuste $(\mathrm{GFI}=.99)$ y estadístico de bondad de ajuste corregido (AGFI $=.99)$ mayores a .90 . 
Asimismo, la raíz del error cuadrático de los residuos $<.07$ fue adecuado $(\mathrm{RMRS}=.048)$, según al criterio de Kelley (1935).

Al igual que Matthey (2011) se compararon los grupos de mujeres, por lo que se encontraron diferencias significativas $(U=-.43, p=.001)$ en variable experiencia del embarazo con un tamaño del efecto mediano (d $=.56$ ). Con ello se determina que las primerizas perciben negativamente la experiencia del embarazo en mayor medida que las multigestas y se encuentran más insatisfechas con el rol materno. Aunque en las variables de estrés percibido y autoeficacia no se muestran diferencias entre grupos (Tabla 5).

\section{Tabla 5}

Diferencias por Número de Embarazo en las Variables de Estudio

\begin{tabular}{|c|c|c|c|c|c|c|c|}
\hline \multirow[t]{2}{*}{ Variables } & $\begin{array}{l}\text { Primigestas } \\
(n=199)\end{array}$ & & $\begin{array}{l}\text { Multigestas } \\
(n=101)\end{array}$ & & \multicolumn{2}{|c|}{$\begin{array}{l}\text { U de Mann } \\
\text { Whitney }\end{array}$} & \\
\hline & Media & $\mathrm{DE}$ & Media & $\mathrm{DE}$ & Z & $p$ & $\mathrm{~d}$ \\
\hline $\begin{array}{l}\text { Experiencia emocional del } \\
\text { embarazo }^{a}\end{array}$ & 10.69 & 7.43 & 6.86 & 5.97 & -.431 & .001 & .56 \\
\hline Estrés percibido & 18.85 & 7.13 & 19.87 & 7.60 & -.40 & .968 & -.13 \\
\hline Autoeficacia & 32.32 & 6.40 & 33.42 & 6.47 & -1.75 & .080 & -.17 \\
\hline
\end{tabular}

Nota: $a=$ instrumento con 9 ítems. $\mathrm{DE}=$ desviación estándar. $\mathrm{p}=$ significancia asintótica bilateral. $\mathrm{d}$ $=$ tamaño del efecto.

Finalmente, se encontró que la pregunta Si has sentido que ser madre es muy estresante, muy difícil o desagradable ¿A qué crees que se deba?, fue respondida por el $21.33 \%(n=64)$ de la muestra total. De las cuales, el 76.57\% $(n=49)$ fueron primerizas y el $23.43 \%(n=15)$ multigestas, e informaron datos cualitativos importantes. Dentro del primer grupo, se 
identificó que el 89.79\% $(\mathrm{n}=44)$ mostró puntuaciones $\geq 9$; indicándose que la maternidad era estresante o difícil debido a que se trataba del primer embarazo; por falta de experiencia, información y preparación; por las nuevas responsabilidades; miedo a no ser buena madre; dificultades económicas; cambios corporales y de humor; embarazo no deseado y por el proyecto de vida inconcluso. En cuanto a las madres multigestas, se encontró que el $73.3 \%(n=11)$ obtuvo valores $\geq 9$, indicándose que el estrés o las dificultades iban en función del número de hijos y la crianza; complicaciones en partos previos; dificultades de pareja y la edad al momento de la evaluación.

\section{DISCUSIÓN}

El objetivo del presente estudio fue evaluar la estructura interna de la escala Being a Mother en su adaptación al español en una muestra de mujeres mexicanas embarazadas, como medida de la experiencia emocional del embarazo y satisfacción con el rol materno. Así, mediante el AFE se determina una estructura unidimensional mediante evidencias de validez de criterio, consistencia interna e indicadores de bondad de ajuste arrojados por el método de Hull. La fiabilidad reportada es similar a la obtenida en el estudio de Henshaw et al. (2014) en una muestra de mujeres primigestas $(\alpha=.84)$ y por Göbel et al. (2020) $(\alpha=.82)$. Además resultó superior a la obtenida por Matthey (2011) $(\alpha=.79)$; sin embargo, no se logró reproducir la estructura de 3 factores, al identificarse reactivos con bajo desempeño para la muestra de mujeres embarazadas mexicanas. Se hipotetiza que esto es atribuible a un factor de deseabilidad social, ya que la mayoría de las participantes optó por elegir respuestas favorables en los reactivos eliminados. Aunque, cabe la posibilidad que éstos resultaran confusos por su redacción o longitud, como en el caso de los ítems 8 y 10. 
Como se trató anteriormente, si bien los ítems del instrumento muestran una buena confiabilidad, la correlación total de elementos corregida en los ítems 1, 4, 8 y 10 detecta una baja correlación entre las preguntas, lo que permite plantear que éstos miden un constructo distinto o son poco relevantes para el instrumento. Incluso se sugiere que la redacción en dirección positiva de los tres primeros ítems, pudo haber influido en lo anterior, pero al evaluar en conjunto su alfa de Cronbach resulta poco consistente, aunado a ello el análisis factorial no identifica más de una dimensión, determinándose su eliminación.

Respecto al ítem 1, que informa sobre la confianza materna, pudo haberse malinterpretado la palabra confiada al considerarse en términos de seguridad, capacidad u otro aspecto diferente para cada participante. Finalmente en el ítem 4, se observó que las mujeres primerizas exhibieron dudas sobre la forma de responderlo (Me he sentido muy cercana o apegada a mi bebé), comentando no saber qué contestar ya que el bebé aún no había nacido. Matthey (2011) manifiesta que este reactivo trata sobre la vinculación de la madre con el recién nacido o preescolar, y no de un problema común por el que pasan las mujeres durante la crianza o maternidad, por lo que una calificación alta denotaría una problemática emocional a nivel clínico. No obstante, sería importante explorar la vinculación emocional con el bebé en muestras de mujeres embarazadas.

En este sentido, se recomienda utilizar el instrumento unidimensional recurriendo a los 9 ítems que poseen direccionalidad inversa, con un recorrido de respuesta de 0 a 26 , donde igualmente a mayores puntajes corresponde una mayor insatisfacción con la experiencia emocional del embarazo y el rol materno. Cabe mencionar, que el cuestionamiento cualitativo es capaz de arrojar información acerca de las dificultades por 
las que pasa una mujer embarazada y aquellos factores que influyen en el incremento del estrés y percepción de insatisfacción. Incluso, en más de la mitad de las mujeres que emitieron una respuesta escrita, se observa una correspondencia con el punto de corte.

Como era de esperar, se presentaron diferencias estadísticamente significativas entre los grupos respecto a la variable experiencia emocional del embarazo, mostrándose que las mujeres primerizas poseen una vivencia más insatisfactoria con el papel de la maternidad y/o el mismo embarazo. Ello es congruente con el estudio de Matthey (2011), quien dentro de sus hallazgos demostró que la maternidad es más difícil para las primerizas que para las multíparas durante la crianza, reportándose mayor insatisfacción por la falta de confianza, dificultad para lidiar con el llanto del bebé, preocupación por no ser buenas madres y la experimentación del sentimiento de culpa. Así, lo anterior apoya nuestros hallazgos ya que existe mayor proporción de mujeres primerizas por encima del punto de corte, indicativo de una insatisfacción con el embarazo y el rol de madre. Asimismo, los aspectos cualitativos que se arrojaron indican que la etapa resulta estresante por ser la primera experiencia en su vida, lo que supone nuevas responsabilidades y el reconocimiento de la falta de experiencia y preparación, a partir de lo cual se puede derivar el miedo a no ser buena madre. Igualmente intervienen aspectos relacionados con factores sociodemográficos y expectativas relacionadas con el plan de vida.

Finalmente, lo anterior podría representar un riesgo para las mujeres debido a que hallazgos reportan que la presencia de estados emocionales negativos prenatales, así como creencias inadaptadas sobre el rol materno y la crianza desde la etapa de la gestación, pueden repercutir durante el postparto manifestándose en una insatisfacción emocional, percepción 
negativa de la maternidad y exacerbación de síntomas psicológicos (Göbel et al., 2020; Henshaw et al., 2014).

En cuanto al estrés percibido y la autoeficacia, no se muestran diferencias significativas, concluyéndose que ambos grupos los experimentan de forma similar y que finalmente existen otros factores de riesgo que influyen en la presencia de la insatisfacción emocional del embarazo y el rol materno. Por su parte, la autoeficacia materna es un proceso dinámico y cambiante que puede disminuir cuando la madre se percibe con pocos recursos para enfrentar las demandas de la maternidad y la crianza, trayendo a su vez síntomas psicopatológicos (Bandura, 1989). Por otra, las participantes se encontraban sanas y sin complicaciones obstétricas al momento del estudio, atribuyéndose a ello un menor estrés percibido (Peñacoba-Puente et al., 2012). Sin embargo, conocer lo anterior queda fuera del alcance del presente estudio.

Por otro lado, las medidas de validez de criterio mostraron una asociación positiva y significativa entre la experiencia del embarazo y el estrés percibido, asimismo, una asociación negativa y significativa entre la experiencia del embarazo y autoeficacia. Esto es congruente con estudios que señalan que altos niveles de estrés percibido, se relacionan con interpretaciones inadecuadas del embarazo (Fleuriet \& Sunil, 2016). Además, que los juicios de incapacidad, contribuyen a la sensación de pérdida de control ante estresores, afectando el estado de ánimo de mujeres embarazadas (Kunseler et al., 2014). Lo anterior se relaciona con Bandura (1989) quien indica que la autoeficacia es un recurso cognitivo que puede afectar la cantidad de estrés, depresión y niveles de motivación frente a situaciones amenazantes o difíciles. 
Como limitaciones del estudio, contemplamos la falta de representatividad de la muestra; asimismo, los datos recogidos comprenden una parte de la población socialmente vulnerable, recomendándose abarcar otros sectores sociodemográficos. Los instrumentos utilizados son medidas de autoinforme y probablemente en algunos ítems se enfrentó una situación de deseabilidad social. Aunado a esto, la experiencia emocional evaluada corresponde al embarazo, por lo que se toma en cuenta que cada etapa de la maternidad posee vivencias diferentes (Matthey, 2011). No obstante, la versión adaptada es capaz de informar la satisfacción con el rol materno y la experiencia acontecida durante la gestación.

\section{CONCLUSIONES}

En conclusión, la versión adaptada al español de la escala Being a Mother para mujeres embarazadas, posee adecuadas propiedades psicométricas y los hallazgos deben tomarse como complemento del trabajo de Matthey (2011). Finalmente, los resultados pueden tener importantes implicaciones prácticas ya que la experiencia del embarazo explica un porcentaje importante de la varianza, lo que permite concluir que esta variable es de suma importancia en la atención prenatal. Por un lado, su exploración mediante un instrumento breve, válido y confiable puede contribuir con la identificación de factores psicosociales asociados a la insatisfacción de las mujeres durante el embarazo, que permitan explicar por qué se está evaluando negativamente la etapa o la maternidad y cómo estas vivencias pueden repercutir en un estado de ánimo negativo e incluso afectaciones más graves, tales como la depresión y ansiedad. Por otro, puede contribuir con la implementación de estrategias de prevención y tratamiento que reduzcan riesgos de la salud mental perinatal de la mujer, y en el desarrollo del bebé. 


\section{Conflicto de intereses}

No existe ningún conflicto de interés.

\section{Agradecimientos}

Las autoras agradecen a la Secretaría de Salud del Estado de Coahuila, México, por las facilidades brindadas para la implementación del estudio. Así como a la Mtra. Maritza Delgado Herrada por sus valiosos comentarios al manuscrito.

\section{REFERENCIAS}

I Ajinkya, S., Jadhav, P. R., \& Srivastava, N. N. (2013). Depression during pregnancy: prevalence and obstetric risk factors among pregnant women attending a tertiary care hospital in Navi Mumbai. Industrial Psychiatry Journal, 22(1), 37-40. https://doi.org/10.4103/0972-6748.123615

I American Psychological Association. (2017). Ethical principles of psychologists and code of conduct. Washington DC: APA, American Psychological Association. Online assessment measures. Disponible en: https://www.apa.org/ethics/code/ethics-code-2017.pdf.

I Ato, M., López, J. J., \& Benavente, A. (2013). Un sistema de clasificación de los diseños de investigación en psicología. Anales de Psicología, 29(3), 1038-59. https://doi.org/10.6018/analesps.29.3.178511

I Baessler, J. \& Schwarcer, R. (1996). Evaluación de la autoeficacia: Adaptación española de la escala de Autoeficacia General. Ansiedad y Estrés, 2, 1-8.

I Bandura, A. (1989). Regulation of cognitive processes through perceived self-efficacy. Developmental Psychology, 25, 725-739. https://doi.org/10.1037/0012-1649.25.5.729

1 Camberis, A. L., McMahon, C. A., Gibson, F. L., \& Boivin, J. (2014). Age, psychological maturity, and the transition to motherhood among English-speaking Australian women in a metropolitan area. Developmental Psychology, 50(8), 2154-2164. https://doi.org/10.1037/a0037301

I Carretero-Dios, H. \& Pérez, C. (2005). Normas para el desarrollo y revisión de estudios instrumentales. International Journal of Clinical and Health Psychology, 5(3), 521-551. Disponible en: https://www. redalyc.org/pdf/337/33705307.pdf

I Cohen, S., Kamarck, T., \& Mermelstein, R. (1983). A global measure of perceived stress. Journal of Health and Social Behavior, 24(4), 385-396. https://doi.org/10.2307/2136404

I Condon J. T. (1993). The assessment of antenatal emotional attachment: Development of a questionnaire instrument. The British Journal of Medical Psychology, 66(Pt 2), 167-183. https://doi. org/10.1111/j.2044-8341.1993.tb01739.x

$\checkmark$ Cox, J. L., Holden, J. M., \& Sagovsky, R. (1987). Detection of postnatal depression. Development of the 10-item Edinburgh Postnatal Depression Scale. The British Journal of Psychiatry, 150(6), 782-786. https:// doi.org/10.1192/bjp.150.6.782 
I Clark-Carter, D. (2002). Investigación cuantitativa en psicología. Oxford University Press.

1 Crncec, R., Barnett, B., \& Matthey, S. (2008). Development of an instrument to assess perceived selfefficacy in the parents of infants. Research in Nursing \& Health, 31(5), 442-453. https://doi.org/10.1002/ nur.20271

I Crncec, R., Barnett, B., \& Matthey, S. (2010). Review of scales of parenting confidence. Journal of Nursing Measurement, 18(3), 210-240. https://doi.org/10.1891/1061-3749.18.3.210

I Ferrando, P. J. \& Anguiano-Carrasco, C. (2010). El análisis factorial como técnica de investigación en psicología. Papeles del Psicólogo, 31(1), 18-33. Disponible en: https://www.redalyc.org/ pdf/778/77812441003.pdf

I Fleuriet, K. J. \& Sunil, T. S. (2016). Stress, pregnancy, and motherhood: Implications for birth weights in the borderlands of Texas. Medical Anthropology Quarterly, 31(1), 1-18. https://doi.org/10.1111/maq.12324

I Frias-Navarro, D. (2020). Apuntes de consistencia interna de las puntuaciones de un instrumento de medida. Universidad de Valencia. Disponible en: https://www.uv.es/friasnav/AlfaCronbach.pdf

$\checkmark$ Göbel, A., Stuhrmann, L. Y., Barkmann, C., Schulte-Markwort, M., \& Mudra, S. (2020). Becoming a mother: Predicting early dissatisfaction with motherhood at three weeks postpartum. Midwifery, 91, 102824. https://doi.org/10.1016/j.midw.2020.102824

$\checkmark$ Gómez, M. E. \& Aldana, E. (2007). Alteraciones en la mujer con embarazo de alto riesgo. Psicología y Salud, 17(001), 53-61. Disponible en: https://www.redalyc.org/pdf/291/29117106.pdf

$\checkmark$ González, M. T. \& Landero, R. (2007). Factor structure of perceived stress scale (PSS) in a sample from Mexico. The Spanish Journal of Psychology, 10(1), 199-206. https://doi.org/10.1017/s1138741600006466

I Hair, J. F., Black, W. C., Babin, B. J., \& Anderson, R. E. (2014). Multivariate data analysis (7 ed.). Pearson.

I Henshaw, E. J., Fried, R., Teeters, J. B., \& Siskind, E. E. (2014). Maternal expectations and postpartum emotional adjustment in first-time mothers: Results of a questionnaire survey. Journal of Psychosomatic Obstetrics \& Gynecology, 35(3), 69-75. https://doi.org/10.3109/0167482x.2014.937802

$1 \mathrm{Hu}$, L. \& Bentler, P. M. (1999). Cutoff criteria for fit indexes in covariance structure analysis: Conventional criteria versus new alternatives. Structural Equation Modeling: A Multidisciplinary Journal, 6(1), 1-55. https://doi.org/10.1080/10705519909540118

$\checkmark$ Jadresic, E. (2014). Depresión perinatal: Detección y tratamientos. Revista Médica Clínica Las Condes, 25(6), 1019-1028. https://doi.org/10.1016/S0716-8640(14)70651-0

I Kelley, T. L. (1935). Essential traits of mental life: The purposes and principles underlying the selection and measurement of independent mental factors, together with computational tables. Harvard University Press.

I Kunseler, F. C., Willemen, A. M., Oosterman, M., \& Schuengel, C. (2014). Changes in parenting selfefficacy and mood symptoms in the transition to parenthood: A bidirectional association. Parenting. Science and Practice, 14(3-4), 215-234. https://doi.org/10.1080/15295192.2014.972758 
1 Lara, M. A. \& Navarrete, L. (2012). Detección de depresión en mujeres embarazadas mexicanas con la CES-D. Salud Mental, 35(1), 57-61. Disponible en: https://www.medigraphic.com/cgi-bin/new/resumen. cgi?IDARTICULO=33547

I Lorenzo-Seva, U. \& Ferrando, P. J. (2013). FACTOR 9.2: A comprehensive program for fitting exploratory and semiconfirmatory factor analysis and IRT models. Applied Psychological Measurement, 37(6), $497-$ 498. https://doi.org/10.1177/0146621613487794

I Lloret-Segura, S., Ferreres-Traver, A., Hernández-Baeza, A., \& Tomás-Marco, I. (2014). El análisis factorial exploratorio de los ítems: Una guía práctica, revisada y actualizada. Anales de Psicología, 30(3), 1151-1169. https://doi.org/10.6018/analesps.30.3.199361

I Lorenzo-Seva, U., Timmerman, M. E., \& Kiers, H. A. L. (2011). The Hull method for selecting the number of common factors. Multivariate Behavioral Research, 46(2), 340-364. https://doi.org/10.1080/00273171 .2011 .564527

1 Matthey, S. (2009). Women's perceptions of the causes of their postnatal distress: Development of the reasons for postnatal distress checklist. Depression and Anxiety, 26(10), 938-948. https://doi.org/10.1002/ da. 20485

1 Matthey, S. (2011). Assessing the experience of motherhood: The being a mother scale (BAM-13). Journal of Affective Disorders, 128, 142-152. https://doi.org/10.1016/j.jad.2010.06.032

$\checkmark$ Nunnally, J. C. (2009). Teoría psicométrica (1 $1^{\mathrm{a}}$ ed.). Trillas.

I Peñacoba-Puente, C., Carmona-Monge, F. J., Marín-Morales, D., \& Naber, K. (2012). Coping strategies of Spanish pregnant women and their impact on anxiety and depression. Research in Nursing \& Health, 36(1), 54-64. https://doi.org/10.1002/nur.21513

I Sandín, B. \& Chorot, P. (2017). Cuestionario de sucesos vitales (CVS): Estructura factorial, propiedades psicométricas y datos normativos. Revista de Psicopatología y Psicología Clínica, 22(2), 95-15. https:// doi.org/10.5944/rppc.vol.22.num.2.2017.19729

I Schwarzer, R. \& Jerusalem, M. (1995). Generalized Self-Efficacy scale. In J. Weinman, S. Wright, \& M. Johnston, Measures in health psychology: A user's portfolio. Causal and control beliefs (pp. 35-37). Windsor, England: NFER-NELSON. Disponible en: http://userpage.fu-berlin.de/ health/faq gse.pdf

I Soma-Pillay, P., Nelson-Piercy, C., Tolppanen, H., \& Mebazaa, A. (2016). Physiological changes in pregnancy. Cardiovascular Journal of Africa, 27(2), 89-94. https://doi.org/10.5830/CVJA-2016-021

I Taubman-Ben-Ari, O., Ben-Shlomo, S., Sivan, E., \& Dolizki, M. (2009). The transition to motherhood: A time for growth. Journal of Social and Clinical Psychology, 28(8), 943-970. https://doi.org/10.1521/ jscp.2009.28.8.943

I Timmerman, M. E. \& Lorenzo-Seva, U. (2011). Dimensionality assessment of ordered polytomous items with parallel analysis. Psychological Methods, 16(2), 209-220. https://doi.org/10.1037/a0023353

I Valdés, M. \& García- Esteve, L1. (2017). Estrés prenatal materno. En L1. García-Esteve, y M. Valdés (Eds.), Manual de psiquiatría perinatal. Guía para el manejo de los trastornos mentales durante el embarazo, postparto y lactancia (pp. 43-55). Panamericana. 
I World Medical Association. (2013). World medical association declaration of Helsinki: Ethical principles for medical research involving human subjects. Journal of the American Medical Association, 310(20), 2191-2194. https://doi.org/10.1001/jama.2013.281053

Envío a dictamen: 8 de abril de 2021

Reenvío: 26 de abril de 2021 Aprobación: 18 de mayo de 2021

Mónica Reyna Martínez. Maestra en Psicología. Candidata a Doctora en Filosofía con orientación en Psicología, Facultad de Psicología, Universidad Autónoma de Nuevo León. Su trabajo se centra en el estudio de variables relacionadas con la psicología de la salud, intervenciones para el afrontamiento y disminución de sintomatología emocional. Docente de Asignatura, Facultad de Psicología, Universidad Autónoma de Coahuila. Correo electrónico: monica.reymar@gmail.com

Minerva T. Vanegas Farfano. PhD. en Psicología. Investigadora Nacional Nivel I (SNI I). Su trabajo se centra en la salud mental y los pasatiempos e intervenciones breves para la regulación emocional y el estrés. Docente de Tiempo Completo, Facultad de Organización Deportiva, Universidad Autónoma de Nuevo León; Monterrey, Nuevo León, México. Correo electrónico: minerva.vanegas@gmail.com

Mónica T. González-Ramírez. Doctora en Psicología. Investigadora Nacional Nivel II (SNI II). Su trabajo se centra en el estudio del estrés y otras variables relacionadas a la salud. Cuerpo Académico en Psicología Social y de la Salud, Facultad de Psicología, Universidad Autónoma de Nuevo León. Correo electrónico: monica.gonzalezrz@uanl. edu.mx,monygzz77@yahoo.com 